\title{
Numerical investigation of advection-diffusion in an inhomogeneous medium with a thin channel using the multiscale finite element method
}

\author{
Mazuriak N. V., Savula Ya. H. \\ Ivan Franko National University of Lviv, \\ 1 Universytetska Str., 79000, Lviv, Ukraine
}

(Received 19 November 2019; Revised 12 May 2020; Accepted 13 May 2020)

\begin{abstract}
The advection-diffusion in an inhomogeneous medium with a thin channel is considered. The multiscale finite element method is applied to solving the formulated model problem. It is shown that the obtained solution is stable and convergent for sufficiently large Peclet numbers. Numerical examples are presented and analysed.
\end{abstract}

Keywords: multiscale finite element method, advection-diffusion, inhomogeneous medium.

2010 MSC: $65 \mathrm{~N} 55,65 \mathrm{~N} 30,80 \mathrm{M} 25,80 \mathrm{~A} 20$

DOI: $10.23939 / \mathrm{mmc} 2020.01 .146$

\section{Introduction}

A large number of heat and mass transfer processes are characterized by the availability of a complex, inhomogeneous structure of the medium, that often contains homogeneous thin inclusions. Solving the advection-diffusion problems in such mediums requires a lot of computational costs using standard numerical methods including the finite element method (FEM) [1]. On the other hand, inaccurate and even inappropriate results may be obtained without taking into account the small scale features of the medium. Besides this, solving the advection-diffusion problem by FEM in the case of large Peclet numbers becomes highly complicated because of the instability of the computational process.

The multiscale finite element method (MsFEM) $[2,3]$ was designed for solving the diffusion problems in an inhomogeneous medium. It captures correctly the large scale components on the course grid without accurately resolving all the small scale features of the solution. This effect is achieved by incorporating the local microstructures of the differential operator into the multiscale basis functions. It was shown [4-7] that MsFEM is also efficient for solving the advection-diffusion problems with large Peclet numbers. In $[8,9]$ the advection-diffusion problem in the homogeneous medium with a thin curvilinear channel was considered. The two-dimensional equation in the channel was reduced to the one-dimensional case and the bubble functions FEM was applied to solving the obtained problem. In this paper MsFEM is applied to solving the advection-diffusion problems with sufficiently large Peclet numbers in an inhomogeneous medium with a thin channel.

\section{Formulation of the problem}

We consider an inhomogeneous medium with a thin channel (see Fig. 1). Let us construct the curvilinear coordinate system $\alpha_{1}, \alpha_{2}$, that is related to the middle curve of the channel so coordinate $\alpha_{1}$ corresponds to the direction of curve tangent and coordinate $\alpha_{2}$ corresponds to the direction of curve normal [7-9]. We suppose the middle curve is smooth and is defined by the parametric equation

$$
x_{1}=x_{1}\left(\alpha_{1}\right), \quad x_{2}=x_{2}\left(\alpha_{1}\right) .
$$


Let us denote by $A$ the Lame coefficient of curve (1) and by $K$ the curvature of the curve [10]

$$
A=\sqrt{\left(x_{1}^{\prime 2}+x_{2}^{\prime 2}\right)}, \quad K=\frac{x_{2}^{\prime \prime} x_{1}^{\prime}-x_{1}^{\prime \prime} x_{2}^{\prime}}{A^{3}} .
$$

According to [7], the advection-diffusion problem in the thin curvilinear channel is as follows:

$$
\begin{gathered}
-\frac{1}{H} \frac{\partial}{\partial \alpha_{1}}\left(\frac{\lambda}{H} \frac{\partial U}{\partial \alpha_{1}}\right)-\frac{1}{H} \frac{\partial}{\partial \alpha_{2}}\left(\lambda H \frac{\partial U}{\partial \alpha_{2}}\right)+\frac{w}{H} \frac{\partial U}{\partial \alpha_{1}}=f, \quad \alpha_{1}, \alpha_{2} \in \Omega, \\
\frac{\lambda}{H} \frac{\partial U}{\partial \alpha_{1}}=\beta^{b}\left(U-U_{m}^{b}\right) \quad \text { on } \quad \Gamma^{b}, \quad-\frac{\lambda}{H} \frac{\partial U}{\partial \alpha_{1}}=\beta^{e}\left(U-U_{m}^{e}\right) \quad \text { on } \quad \Gamma^{e}, \\
\lambda \frac{\partial U}{\partial \alpha_{2}}=q^{-} \quad \text { on } \quad \Gamma^{-}, \quad-\lambda \frac{\partial U}{\partial \alpha_{2}}=q^{+} \quad \text { on } \quad \Gamma^{+},
\end{gathered}
$$

where $H=A\left(1+\alpha_{2} K\right), U\left(\alpha_{1}, \alpha_{2}\right)$ is the unknown function, $\lambda=$ const $\geqslant 0$ is the diffusion coefficient, $w=$ const is the advection coefficient, $f\left(\alpha_{1}, \alpha_{2}\right)$ is the function that characterizes the intensity of internal sources, $q^{-}\left(\alpha_{1}, \alpha_{2}\right), q^{+}\left(\alpha_{1}, \alpha_{2}\right)$ are the given functions, $U_{m}^{b}, U_{m}^{e}$ are the values on the boundaries $\Gamma^{b}$, $\Gamma^{e}$ respectively.

Let $U_{1}\left(x_{1}, x_{2}\right), U_{2}\left(x_{1}, x_{2}\right)$ be the unknown functions in $\Omega_{1}$, $\Omega_{2}$ respectively and let $2 h$ be the channel thickness. We assume that the channel is thin, that is $h K \ll 1$. Under this assumption the solution in the channel can be presented in the form of linear function of the variable $\alpha_{2}$ [8]

$$
U\left(\alpha_{1}, \alpha_{2}\right)=u_{1}\left(\alpha_{1}\right)+\frac{\alpha_{2}}{h} u_{2}\left(\alpha_{1}\right)
$$

where $u_{1}\left(\alpha_{1}\right), u_{2}\left(\alpha_{1}\right)$ are the unknown functions. Therefore, the advection-diffusion problem (2)-(4) can be reduced to the one-dimensional case [7] and the mathematical model of

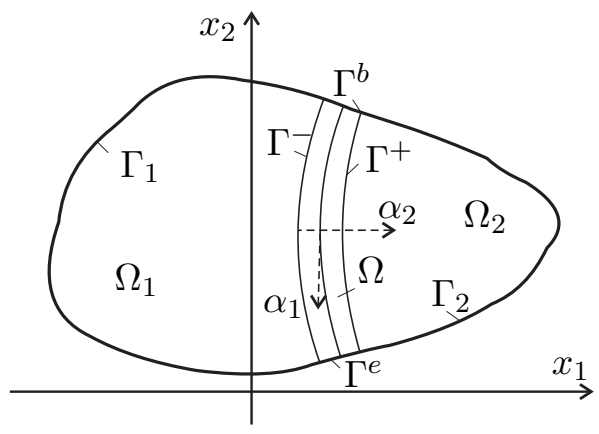

Fig. 1. Medium with a thin channel advection-diffusion in the medium with a thin channel is as follows:

$$
\begin{gathered}
-\frac{1}{A} \frac{\partial}{\partial \alpha_{1}} \frac{\lambda}{A}\left(\frac{\partial u_{1}}{\partial \alpha_{1}}-\frac{h K}{3} \frac{\partial u_{2}}{\partial \alpha_{1}}\right)+\frac{1}{A} w \frac{\partial u_{1}}{\partial \alpha_{1}} \\
=\frac{1}{2 h}\left(\int_{-h}^{h} f\left(1+\alpha_{2} K\right) d \alpha_{2}-(1+h K) q^{+}-(1-h K) q^{-}\right), \quad \alpha_{1}, \alpha_{2} \in \Omega, \\
-\frac{1}{A} \frac{\partial}{\partial \alpha_{1}} \frac{\lambda}{A}\left(\frac{\partial u_{2}}{\partial \alpha_{1}}-h K \frac{\partial u_{1}}{\partial \alpha_{1}}\right)+\frac{1}{A} w \frac{\partial u_{2}}{\partial \alpha_{1}}+\frac{3 \lambda}{h^{2}} u_{2} \\
=\frac{3}{2 h}\left(\frac{1}{h} \int_{-h}^{h} f\left(1+\alpha_{2} K\right) \alpha_{2} d \alpha_{2}-(1+h K) q^{+}+(1-h K) q^{-}\right), \quad \alpha_{1}, \alpha_{2} \in \Omega, \\
--\nabla \cdot\left(\lambda_{1} \nabla U_{1}\right)+\mathbf{w}_{1} \nabla U_{1}=f_{1}, \quad x_{1}, x_{2} \in \Omega_{1}, \\
\frac{\lambda}{A}\left(\frac{\partial u_{1}}{\partial \alpha_{1}}-\frac{h K}{3} \frac{\partial u_{2}}{\partial \alpha_{1}}\right)=\beta^{b}\left(u_{1}-u_{1}^{b}\right), \quad \frac{\lambda}{A}\left(\frac{\partial u_{2}}{\partial \alpha_{1}}-h K \frac{\partial u_{1}}{\partial \alpha_{1}}\right)=\beta^{b}\left(u_{2}-u_{2}^{b}\right) \quad \text { on } \quad \Gamma^{b}, \\
-\frac{\lambda}{A}\left(\frac{\partial u_{1}}{\partial \alpha_{1}}-\frac{h K}{3} \frac{\partial u_{2}}{\partial \alpha_{1}}\right)=\beta^{e}\left(u_{1}-u_{1}^{e}\right), \quad-\frac{\lambda}{A}\left(\frac{\partial u_{2}}{\partial \alpha_{1}}-h K \frac{\partial u_{1}}{\partial \alpha_{1}}\right)=\beta^{e}\left(u_{2}-u_{2}^{e}\right) \quad \text { on } \quad \Gamma^{e},
\end{gathered}
$$




$$
\begin{gathered}
\lambda_{1} \frac{\partial U_{1}}{\partial n}=\beta_{1}\left(U_{1}-U_{1}^{m}\right) \quad \text { on } \quad \Gamma_{1}, \quad \lambda_{2} \frac{\partial U_{2}}{\partial n}=\beta_{2}\left(U_{2}-U_{2}^{m}\right) \quad \text { on } \quad \Gamma_{2}, \\
U_{1}=u_{1}-u_{2}, \quad \lambda_{1} \frac{\partial U_{1}}{\partial \alpha_{2}}=q^{-} \quad \text { on } \quad \Gamma^{-}, \quad U_{2}=u_{1}+u_{2}, \quad-\lambda_{2} \frac{\partial U_{2}}{\partial \alpha_{2}}=q^{+} \quad \text { on } \Gamma^{+} .
\end{gathered}
$$

Here $\lambda_{i}\left(x_{1}, x_{2}\right)$ are the diffusion coefficients, $\mathbf{w}_{i}=\left\{w_{i 1}, w_{i 2}\right\}$ are vectors that characterize the speed of the advective transfer, $w_{i j}=$ const are the advection coefficients, $f_{i}\left(x_{1}, x_{2}\right)$ are functions that characterize the intensity of internal sources, $U_{i}^{m}$ are the values on the boundaries $\Gamma_{i}, i, j=\overline{1,2}$; $u_{1}^{k}=\frac{1}{2 h} \int_{-h}^{h} U_{m}^{k} d \alpha_{2}, u_{2}^{k}=\frac{3}{2 h^{2}} \int_{-h}^{h} U_{m}^{k} \alpha_{2} d \alpha_{2}, k=b, e$.

\section{Variational formulation}

We consider spaces

$$
\begin{gathered}
V=\left\{v\left(\alpha_{1}\right) \mid v\left(\alpha_{1}\right) \in W_{2}^{(1)}\left(\alpha_{1}^{b}, \alpha_{1}^{e}\right)\right\}, \\
V_{k}=\left\{v\left(x_{1}, x_{2}\right) \mid v\left(x_{1}, x_{2}\right) \in W_{2}^{(1)}\left(\Omega_{k}\right)\right\}, \quad k=\overline{1,2}
\end{gathered}
$$

and bilinear and linear forms

$$
\begin{aligned}
& a^{1}\left(U, \tilde{u}_{1}\right)=-\int_{\alpha_{1}^{b}}^{\alpha_{1}^{e}} 2 h \frac{\partial}{\partial \alpha_{1}} \frac{\lambda}{A}\left(\frac{\partial u_{1}}{\partial \alpha_{1}}-\frac{h K}{3} \frac{\partial u_{2}}{\partial \alpha_{1}}\right) \tilde{u}_{1} d \alpha_{1}, \\
& a^{2}\left(U, \tilde{u}_{2}\right)=-\int_{\alpha_{1}^{b}}^{\alpha_{1}^{e}} \frac{2 h}{3} \frac{\partial}{\partial \alpha_{1}} \frac{\lambda}{A}\left(\frac{\partial u_{2}}{\partial \alpha_{1}}-h K \frac{\partial u_{1}}{\partial \alpha_{1}}\right) \tilde{u}_{2} d \alpha_{1}
\end{aligned}
$$

$$
\begin{gathered}
b^{1}\left(U, \tilde{u}_{1}\right)=\int_{\alpha_{1}^{b}}^{\alpha_{1}^{e}} 2 h w \frac{\partial u_{1}}{\partial \alpha_{1}} \tilde{u}_{1} d \alpha_{1}, \quad b^{2}\left(U, \tilde{u}_{2}\right)=\int_{\alpha_{1}^{b}}^{\alpha_{1}^{e}} \frac{2 h}{3} w \frac{\partial u_{2}}{\partial \alpha_{1}} \tilde{u}_{2} d \alpha_{1}, \quad c^{2}\left(U, \tilde{u}_{2}\right)=\int_{\alpha_{1}^{b}}^{\alpha_{1}^{e}} \frac{2 \lambda}{h} u_{2} \tilde{u}_{2} A d \alpha_{1}, \\
l^{1}\left(\tilde{u}_{1}\right)=\int_{\alpha_{1}^{b}}^{\alpha_{1}^{e}}\left(\int_{-h}^{h} f\left(1+\alpha_{2} K\right) d \alpha_{2}\right) \tilde{u}_{1} A d \alpha_{1}-\int_{\alpha_{1}^{b}}^{\alpha_{1}^{e}}\left((1+h K) q^{+}+(1-h K) q^{-}\right) \tilde{u}_{1} A d \alpha_{1}, \\
l^{2}\left(\tilde{u}_{2}\right)=\int_{\alpha_{1}^{b}}^{\alpha_{1}^{e}} \frac{1}{h}\left(\int_{-h}^{h} f\left(1+\alpha_{2} K\right) \alpha_{2} d \alpha_{2}\right) \tilde{u}_{2} A d \alpha_{1}-\int_{\alpha_{1}^{b}}^{\alpha_{1}^{e}}\left((1+h K) q^{+}-(1-h K) q^{-}\right) \tilde{u}_{2} A d \alpha_{1}, \\
a_{k}\left(U_{k}, \tilde{U}_{k}\right)=-\iint_{\Omega_{k}} \nabla \cdot\left(\lambda_{k} \nabla U_{k}\right) \tilde{U}_{k} d x_{1} d x_{2}, \quad b_{k}\left(U_{k}, \tilde{U}_{k}\right)=\iint_{\Omega_{k}} \mathbf{w}_{k} \nabla U_{k} \tilde{U}_{k} d x_{1} d x_{2}, \\
l_{k}\left(\tilde{U}_{k}\right)=\iint_{\Omega_{k}} f_{k} \tilde{U}_{k} d x_{1} d x_{2}, \quad k=\overline{1,2},
\end{gathered}
$$

where $u_{k}, \tilde{u}_{k} \in V, U_{k}, \tilde{U}_{k} \in V_{k}$.

After applying the integration by parts and the Green's formula to bilinear forms $a^{k}\left(U, \tilde{u}_{k}\right)$, $a_{k}\left(U_{k}, \tilde{U}_{k}\right), k=\overline{1,2}$ and using the boundary conditions (9)-(11) we obtain

$$
\begin{gathered}
a^{1}\left(U, \tilde{u}_{1}\right)=\int_{\alpha_{1}^{b}}^{\alpha_{1}^{e}} 2 h \frac{\lambda}{A}\left(\frac{\partial u_{1}}{\partial \alpha_{1}}-\frac{h K}{3} \frac{\partial u_{2}}{\partial \alpha_{1}}\right) \frac{\partial \tilde{u}_{1}}{\partial \alpha_{1}} d \alpha_{1}+\left.2 h \beta^{e}\left(u_{1}-u_{1}^{e}\right) \tilde{u}_{1}\right|_{\Gamma^{e}}+\left.2 h \beta^{b}\left(u_{1}-u_{1}^{b}\right) \tilde{u}_{1}\right|_{\Gamma^{b}}, \\
a^{2}\left(U, \tilde{u}_{1}\right)=\int_{\alpha_{1}^{b}}^{\alpha_{1}^{e}} \frac{2 h}{3} \frac{\lambda}{A}\left(\frac{\partial u_{2}}{\partial \alpha_{1}}-h K \frac{\partial u_{1}}{\partial \alpha_{1}}\right) \frac{\partial \tilde{u}_{2}}{\partial \alpha_{1}} d \alpha_{1}+\left.\frac{2 h}{3} \beta^{e}\left(u_{2}-u_{2}^{e}\right) \tilde{u}_{2}\right|_{\Gamma^{e}}+\left.\frac{2 h}{3} \beta^{b}\left(u_{2}-u_{2}^{b}\right) \tilde{u}_{2}\right|_{\Gamma^{b}}, \\
a_{1}\left(U_{1}, \tilde{U}_{1}\right)=\iint_{\Omega_{1}} \lambda_{1} \nabla U_{1} \nabla \tilde{U}_{1} d x_{1} d x_{2}-\left.\int_{\Gamma_{1}} \beta_{1}\left(U_{1}-U_{1}^{m}\right) \tilde{U}_{1}\right|_{\Gamma_{1}} d \Gamma-\left.\int_{\alpha_{1}^{b}}^{\alpha_{1}^{e}} \lambda_{1} \frac{\partial U_{1}}{\partial \alpha_{2}} \tilde{U}_{1}\left(1+\alpha_{2} K\right)\right|_{\Gamma^{-}} A d \alpha_{1}, \\
a_{2}\left(U_{2}, \tilde{U}_{2}\right)=\iint_{\Omega_{2}} \lambda_{2} \nabla U_{2} \nabla \tilde{U}_{2} d x_{1} d x_{2}-\left.\int_{\Gamma_{2}} \beta_{2}\left(U_{2}-U_{2}^{m}\right) \tilde{U}_{2}\right|_{\Gamma_{2}} d \Gamma+\left.\int_{\alpha_{1}^{b}}^{\alpha_{1}^{e}} \lambda_{2} \frac{\partial U_{2}}{\partial \alpha_{2}} \tilde{U}_{2}\left(1+\alpha_{2} K\right)\right|_{\Gamma^{+}} A d \alpha_{1} .
\end{gathered}
$$

Mathematical Modeling and Computing, Vol. 7, No.1, pp. 146-157 (2020) 
We take into account conditions $\left.\tilde{U}_{1}\right|_{\Gamma^{-}}=\tilde{u}_{1}-\tilde{u}_{2},\left.\tilde{U}_{2}\right|_{\Gamma^{+}}=\tilde{u}_{1}+\tilde{u}_{2}$, conjugation conditions (12) and add together all bilinear and linear forms. Note that some of addends on the common boundaries $\Gamma^{-}$ and $\Gamma^{+}$annihilate each other, so bilinear and linear forms can be written in a simpler form. Therefore, the variational formulation of the problem (5)-(12) is as follows.

Find functions $U_{1} \in V_{1}, U_{2} \in V_{2}, u_{1} \in V, u_{2} \in V$ which satisfy the variational equation

$$
\begin{array}{r}
a_{1}\left(U_{1}, \tilde{U}_{1}\right)+a_{2}\left(U_{2}, \tilde{U}_{2}\right)+a^{1}\left(U, \tilde{u}_{1}\right)+a^{2}\left(U, \tilde{u}_{2}\right)+b_{1}\left(U_{1}, \tilde{U}_{1}\right)+b_{2}\left(U_{2}, \tilde{U}_{2}\right)+b^{1}\left(U, \tilde{u}_{1}\right)+b^{2}\left(U, \tilde{u}_{2}\right) \\
+c^{1}\left(U, \tilde{u}_{1}\right)+c^{2}\left(U, \tilde{u}_{2}\right)=l_{1}\left(\tilde{U}_{1}\right)+l_{2}\left(\tilde{U}_{2}\right)+l^{1}\left(\tilde{u}_{1}\right)+l^{2}\left(\tilde{u}_{2}\right)
\end{array}
$$

and conditions

$$
\forall \tilde{u}_{k} \in V \forall \tilde{U}_{k} \in V_{k}, \quad k=\overline{1,2}, \quad \tilde{U}_{1}=\tilde{u}_{1}-\tilde{u}_{2} \quad \text { on } \quad \Gamma^{-}, \quad \tilde{U}_{2}=\tilde{u}_{1}+\tilde{u}_{2} \quad \text { on } \quad \Gamma^{+},
$$

where $U_{1}=u_{1}-u_{2}$ on $\Gamma^{-}, U_{2}=u_{1}+u_{2}$ on $\Gamma^{+}$,

$$
\begin{gathered}
a^{1}\left(U, \tilde{u}_{1}\right)=\int_{\alpha_{1}^{b}}^{\alpha_{1}^{e}} 2 h \frac{\lambda}{A}\left(\frac{\partial u_{1}}{\partial \alpha_{1}}-\frac{h K}{3} \frac{\partial u_{2}}{\partial \alpha_{1}}\right) \frac{\partial \tilde{u}_{1}}{\partial \alpha_{1}} d \alpha_{1}, \quad a^{2}\left(U, \tilde{u}_{1}\right)=\int_{\alpha_{1}^{b}}^{\alpha_{1}^{e}} \frac{2 h}{3} \frac{\lambda}{A}\left(\frac{\partial u_{2}}{\partial \alpha_{1}}-h K \frac{\partial u_{1}}{\partial \alpha_{1}}\right) \frac{\partial \tilde{u}_{2}}{\partial \alpha_{1}} d \alpha_{1}, \\
b^{1}\left(U, \tilde{u}_{1}\right)=\int_{\alpha_{1}^{b}}^{\alpha_{1}^{e}} 2 h w \frac{\partial u_{1}}{\partial \alpha_{1}} \tilde{u}_{1} d \alpha_{1}, \quad b^{2}\left(U, \tilde{u}_{2}\right)=\int_{\alpha_{1}^{b}}^{\alpha_{1}^{e}} \frac{2 h}{3} w \frac{\partial u_{2}}{\partial \alpha_{1}} \tilde{u}_{2} d \alpha_{1}, \\
c^{1}\left(U, \tilde{u}_{1}\right)=2 h\left(\left.\beta^{b} u_{1} \tilde{u}_{1}\right|_{\Gamma^{b}}+\left.\beta^{e} u_{1} \tilde{u}_{1}\right|_{\Gamma}\right), \quad c^{2}\left(U, \tilde{u}_{2}\right)=\int_{\alpha_{1}^{b}}^{\alpha_{1}^{e}} \frac{2 \lambda}{h} u_{2} \tilde{u}_{2} A d \alpha_{1}+\frac{2 h}{3}\left(\left.\beta^{b} u_{2} \tilde{u}_{2}\right|_{\Gamma^{b}}+\left.\beta^{e} u_{2} \tilde{u}_{2}\right|_{\Gamma^{e}}\right), \\
l^{1}\left(\tilde{u}_{1}\right)=\int_{\alpha_{1}^{b}}^{\alpha_{1}^{e}}\left(\int_{-h}^{h} f\left(1+\alpha_{2} K\right) d \alpha_{2}\right) \tilde{u}_{1} A d \alpha_{1}+2 h\left(\left.\beta^{b} u_{1}^{b} \tilde{u}_{1}\right|_{\Gamma^{b}}+\left.\beta^{e} u_{1}^{e} \tilde{u}_{1}\right|_{\Gamma^{e}}\right), \\
l_{\alpha_{1}^{b}}^{\alpha_{1}^{e}} \frac{1}{h}\left(\int_{-h}^{h} f\left(1+\alpha_{2} K\right) \alpha_{2} d \alpha_{2}\right) \tilde{u}_{2} A d \alpha_{1}+\frac{2 h}{3}\left(\left.\beta^{b} u_{2}^{b} \tilde{u}_{2}\right|_{\Gamma^{b}}+\left.\beta^{e} u_{2}^{e} \tilde{u}_{2}\right|_{\Gamma^{e}}\right), \\
c_{k}\left(U_{k}, \tilde{U}_{k}\right)=\int_{\Gamma_{k}} \beta_{k} U_{k}=\iint_{\Omega_{k}} \lambda_{k} \nabla U_{k} \nabla \tilde{U}_{k} d x_{1} d x_{2}, \quad b_{k}\left(U_{k}, \tilde{U}_{k}\right)=\iint_{\Omega_{k}} \mathbf{w}_{k} \nabla U_{k} \tilde{U}_{k} d x_{1} d x_{2}, \\
l_{k}\left(\tilde{U}_{k}\right)=\iint_{\Omega_{k}} f_{k} \tilde{U}_{k} d x_{1} d x_{2}-\left.\int_{\Gamma_{k}} \beta_{k} U_{k}^{m} \tilde{U}_{k}\right|_{\Gamma_{k}} d \Gamma, \quad k=\overline{1,2} .
\end{gathered}
$$

\section{Multiscale finite element method}

We generalize the equations (5)-(8) into the following advection-diffusion equation

$$
L u=f,
$$

where $\Omega \subset \mathbb{R}^{d}, d=1,2, L u=-\nabla \cdot(\lambda(x) \nabla u)+\mathbf{w} \nabla u, \lambda(x)$ is the diffusion coefficient that characterizes multiscale inhomogeneities of the medium, $\mathbf{w}$ is a vector in $\mathbb{R}^{d}$ that characterizes the speed of the advective transfer, $u(x)$ is the unknown function, $f(x)$ is the given function that characterize the intensity of internal sources, $x \in \mathbb{R}^{d}$.

Let us consider the application of the multiscale finite element method to the generalized equation (14). The main idea of the MsFEM is to construct the special multiscale basis functions. Let $P_{h}$ be a partition of the domain $\Omega$ into finite elements by points $x_{i}, i=\overline{0, n}$. We call this partition the coarse grid and assume that it can be resolved via a finer resolution called the fine grid. Let $\varphi_{i}^{0}$ be 
the piecewise linear (or bilinear in the case $\Omega \subset \mathbb{R}^{2}$ ) functions, which are the nodal basis functions of FEM. The multiscale basis functions $\varphi_{i}$ can be obtained from the condition

$$
L \varphi_{i}=0 \quad \text { in } \quad \mathrm{K}, \quad \varphi_{i}=\varphi_{i}^{0} \quad \text { on } \quad \partial \mathrm{K}, \quad \forall \mathrm{K} \in P_{h}, \quad \mathrm{~K} \subset S_{i}, \quad i=\overline{0, n},
$$

where $S_{i}$ is the support of $\varphi_{i}^{0}, \mathrm{~K}$ is a coarse grid block of the partition $P_{h}$.

The next step of MsFEM is the global formulation, or assembling the global matrix on the coarse grid. We introduce the space

$$
V=\left\{u(x): u(x) \in W_{2}^{(1)}\right\}
$$

and multiply the equation (14) on the arbitrary function $v \in V$. Integrating the result on $\Omega$ we obtain the following variational formulation. Find such function $u(x) \in V$ that satisfies the condition

$$
(L u, v)=(f, v) \quad \forall v \in V .
$$

We take $v=\varphi_{j}^{0}, j=\overline{0, n}$ in (15). Analogously to FEM we look for the solution of (14) in the form

$$
\tilde{u}(x)=\sum_{i=0}^{n} u_{i} \varphi_{i}(x)
$$

and obtain the following system of equations

$$
\mathbf{A u}=\mathbf{b},
$$

where $\mathbf{A}=\left(a_{i j}\right)$ is the global matrix with elements $a_{i j}=\sum_{s=0}^{n} \int_{K_{s}} L \varphi_{i}(x) \varphi_{j}^{0}(x) d x, i, j=\overline{0, n}, \mathbf{b}=\left(b_{j}\right)$ is the vector with elements $b_{j}=\int_{\Omega} f(x) \varphi_{j}^{0}(x) d x, j=\overline{0, n}, \mathbf{u}=\left(u_{i}\right)$ is the vector of unknown values of the function $u$ in nodes $x_{i} \in P_{h}, i=\overline{0, n}$.

Therefore, the small scale information of the inhomogeneous medium and the local properties of the differential operator are incorporated into the multiscale basis functions within each coarse grid element. Then the small scale information is brought to the large scale through the coupling of the global matrix. Thus, an accurate approximation of the solution is obtained on the large scale without resolving the small scale details, and the small scale information is correctly captured due to the multiscale basis functions.

\section{Numerical experiments}

Example 1. We assume that the medium inside the channel is homogeneous, so $\lambda=$ const, and the medium outside the channel is inhomogeneous, multiscale and periodic, so $\lambda_{1}, \lambda_{2}$ depend on the variables $x_{1}, x_{2}$ and some small parameter $\varepsilon$ that determines the small scale of the problem. Let us consider the layered medium shown in Fig. 2. Here $\lambda=1, \lambda_{1}\left(x_{1}, x_{2}\right)=\frac{1}{4+p\left(\sin \left(2 \pi \frac{x_{1}}{\varepsilon}\right)\right)}, \lambda_{2}\left(x_{1}, x_{2}\right)=$ $\frac{1}{4+p\left(\sin \left(2 \pi \frac{x_{2}}{\varepsilon}\right)\right)}, p=3.8, \varepsilon=0.0078$. The speed of the advective transfer is selected so $w_{12}=w_{22}$ and the Peclet number is 150 in the channel and 100 in the inhomogeneous medium. We use the partition to 8 finite elements on the coarse grid in both $x_{1}$ and $x_{2}$ directions in $\Omega_{1} \cup \Omega_{2}$ domain and to 64 finite elements on the fine grid, that is for every coarse grid block.

For simplicity, we take the homogeneous boundary conditions and the squared medium with the rectilinear channel in the middle for our experiments, so $\Omega_{1}=\left[a ; a+\frac{b-a-h_{\text {thin }}}{2}\right] \times[a ; b], \Omega_{2}=[a+$ $\left.\frac{b-a+h_{\text {thin }}}{2} ; b\right] \times[a ; b], \Omega=\left[a+\frac{b-a-h_{\text {thin }}}{2} ; a+\frac{b-a+h_{\text {thin }}}{2}\right] \times[a ; b]$, where $h_{\text {thin }}$ is the channel thickness (we denote by $h$ the size of a coarse grid block). We use the following values of parameters for numerical experiments: $a=0, b=1, h_{\text {thin }}=0.015, f=f_{1}=f_{2}=1$. We take the direction of advection in the medium the same as in the channel (from $a$ to $b$ along the $x_{2}$ axis), so $w_{11}=w_{21}=0$. 

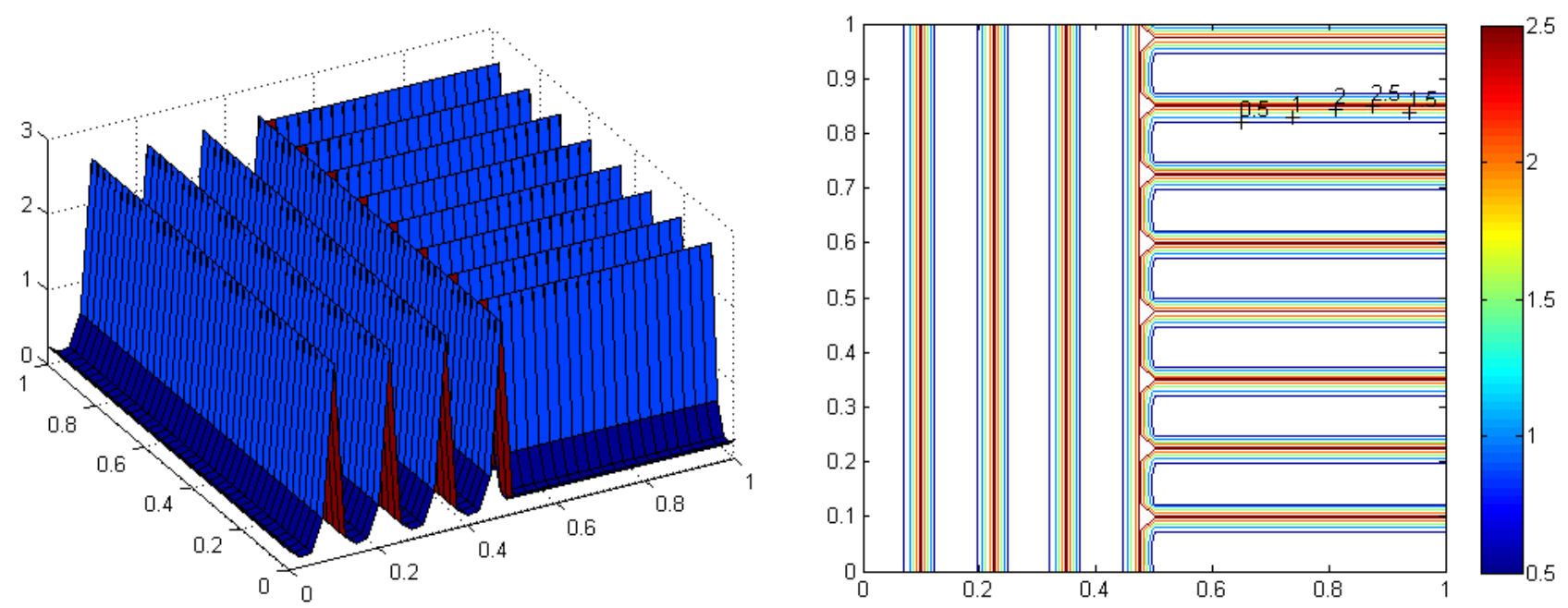

Fig. 2. The layered medium.

In Fig. 3 the multiscale basis functions are presented in $\Omega_{1}$ and $\Omega_{2}$ respectively. As we can see, the multiscale inhomogeneities are incorporated into these basis functions.
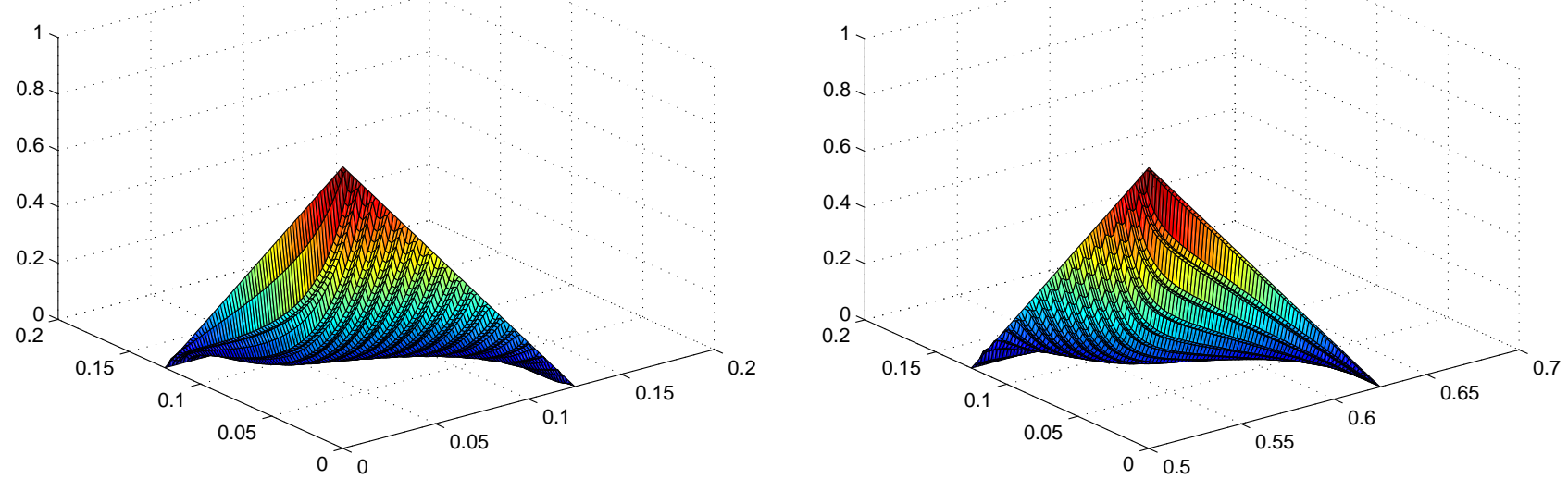

Fig. 3. Basis functions in the layered medium.
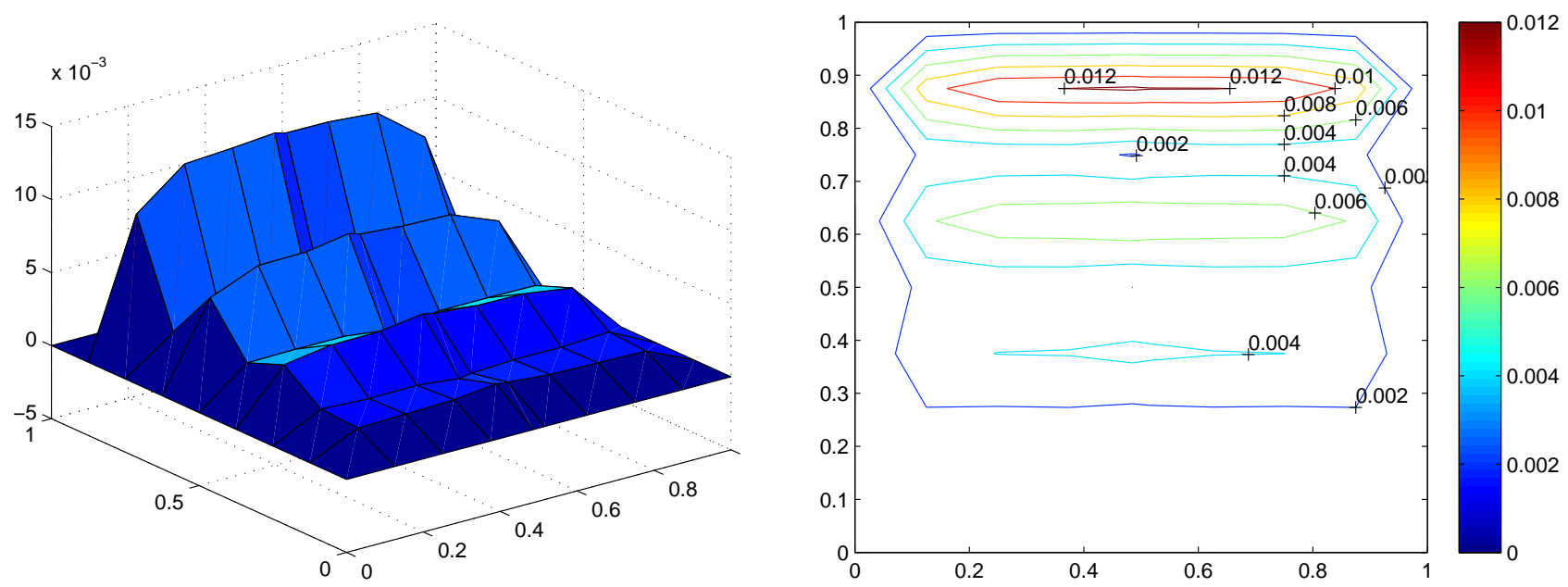

Fig. 4. FEM solution in the layered medium. 
For comparison, the solutions obtained in the whole domain $\Omega_{1} \cup \Omega \cup \Omega_{2}$ by classical and multiscale finite element methods are presented in Figs. 4-5. It should be pointed out that the MsFEM solution is much more stable, while the solution obtained by FEM contains unnatural oscillations. Besides this, the FEM solution behaves in the same way in both multiscale mediums $\Omega_{1}$ and $\Omega_{2}$, and even in the homogeneous channel $\Omega$ with higher Peclet number. The MsFEM solution, in contradistinction to the FEM one, behaves differently in these three mediums and this behaviour corresponds to the natural advection-diffusion process.
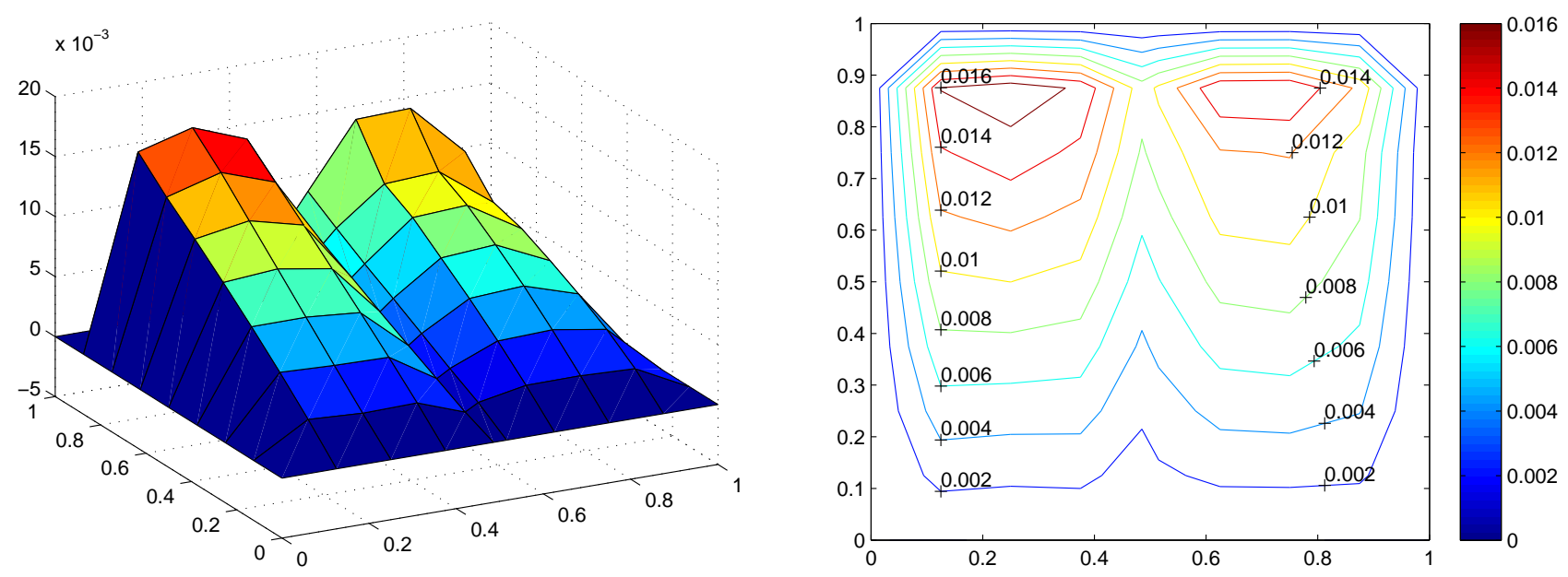

Fig. 5. MsFEM solution in the layered medium.

Example 2. Next we consider the same multiscale medium in both $\Omega_{1}$ and $\Omega_{2}$ with $\lambda_{1}\left(x_{1}, x_{2}\right)=$ $\lambda_{2}\left(x_{1}, x_{2}\right)=\frac{1}{4+p\left(\sin \left(2 \pi \frac{x_{1}}{\varepsilon}\right)+\sin \left(2 \pi \frac{x_{2}}{\varepsilon}\right)\right)}, p=1.9$ shown in Fig. 6 . The speed of the advective transfer here is selected so $w_{12}=w_{22}$ and the Peclet number is 100 in the channel and 70 in the inhomogeneous medium.
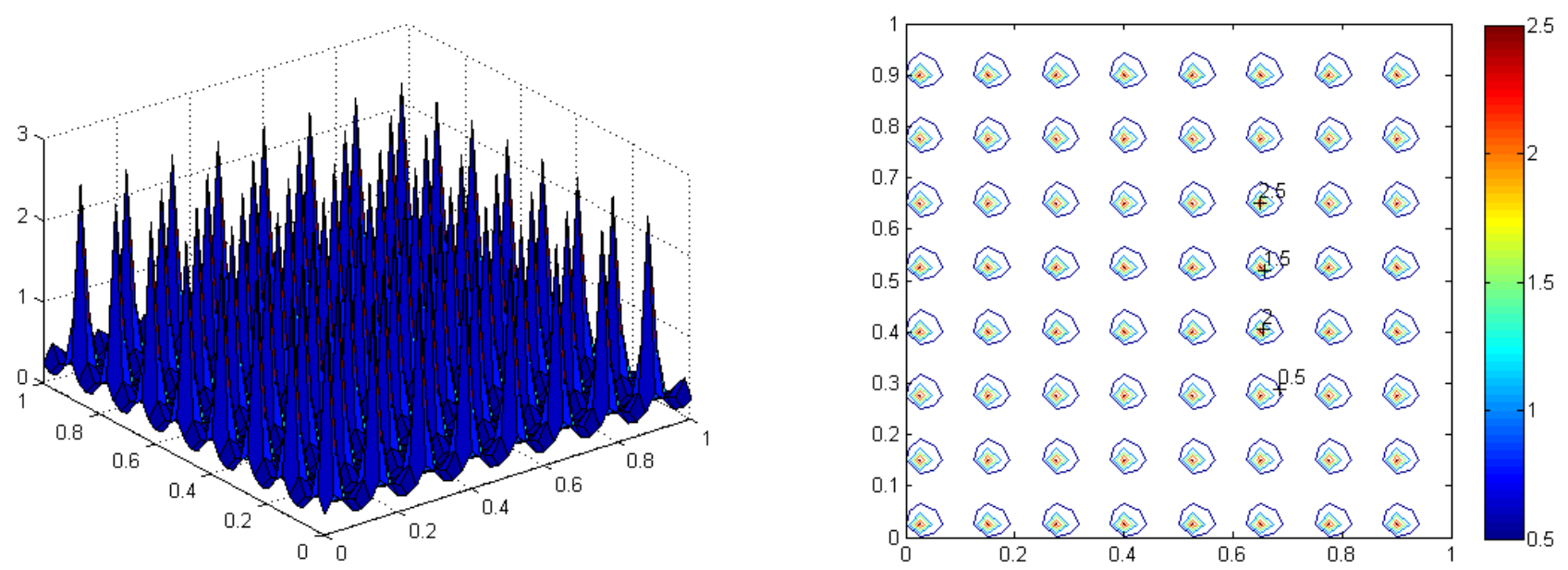

Fig. 6. The multiscale medium.

It was shown in [3] that the MsFEM solution converges to the homogenized solution in the homogenization limit, that is as $\varepsilon \rightarrow 0$. The estimate in the case $h>\varepsilon$ is $\mathrm{O}\left(h+\sqrt{\frac{\varepsilon}{h}}\right)$, where $h$ is the size of a coarse grid element. This estimate is obtained for the diffusion problem, but it is easy to see that it can be obtained also for the advection-diffusion problem in the same way. In order to confirm the given theoretical estimate we use the partition to 4,8 and 16 finite elements in both $x_{1}$ and $x_{2}$ directions on the coarse grid and 16, 32 and 64 finite elements respectively on the fine grid in our 
following numerical experiments. As $\varepsilon$ should be less than the coarse grid element size, grater than the fine grid element size and tend to zero, we use $0.0625,0.0156$ and 0.0039 values for $\varepsilon$, so the ratio $\frac{\varepsilon}{h}$ is $\frac{1}{4}, \frac{1}{8}$ and $\frac{1}{16}$ respectively. All our next experiments are obtained in $\Omega_{1} \cup \Omega \cup \Omega_{2}$ domain.

For comparison, we show the homogeneous results together with the multiscale one, so it is easy to see how the MsFEM solution converges when $\varepsilon \rightarrow 0, h \rightarrow 0$ and $h>\varepsilon$. We take the average value of $\lambda_{i}$, $i=1,2$ in the multiscale medium and use it for obtaining the homogeneous results, so $\lambda_{1}=\lambda_{2}=\lambda_{h}$, $\lambda_{h}=$ const and $\lambda_{h}$ is different for every $\varepsilon$ and independent of $x_{1}$ and $x_{2}$. In Figs.7, 9, 11 the homogeneous and multiscale basis functions are presented for 4, 8 and 16 finite elements respectively. Note that the multiscale basis functions contain the medium inhomogeneities and converge to the appropriate homogeneous basis functions.
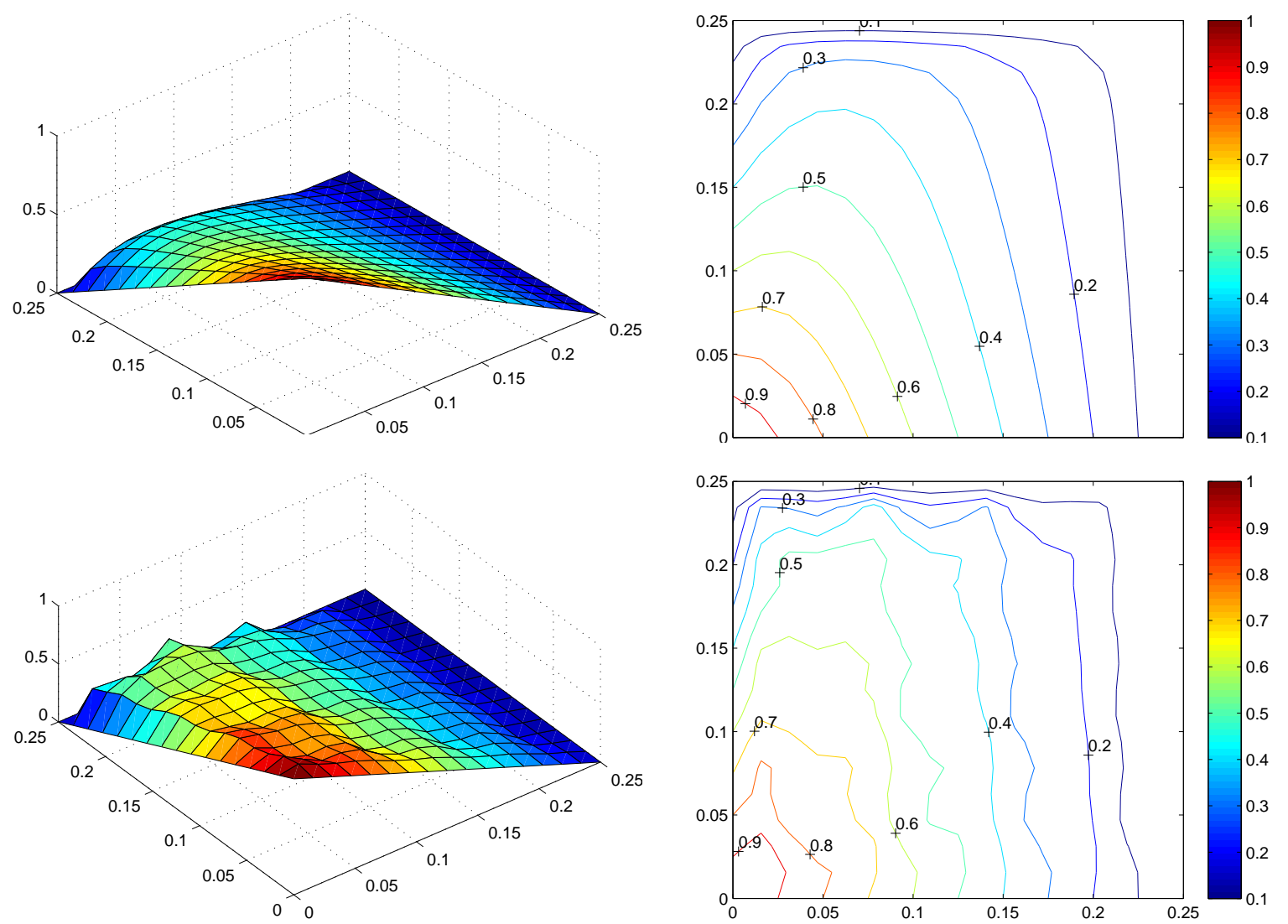

Fig. 7. Homogeneous (top) and multiscale (bottom) basis functions for $n=4$.
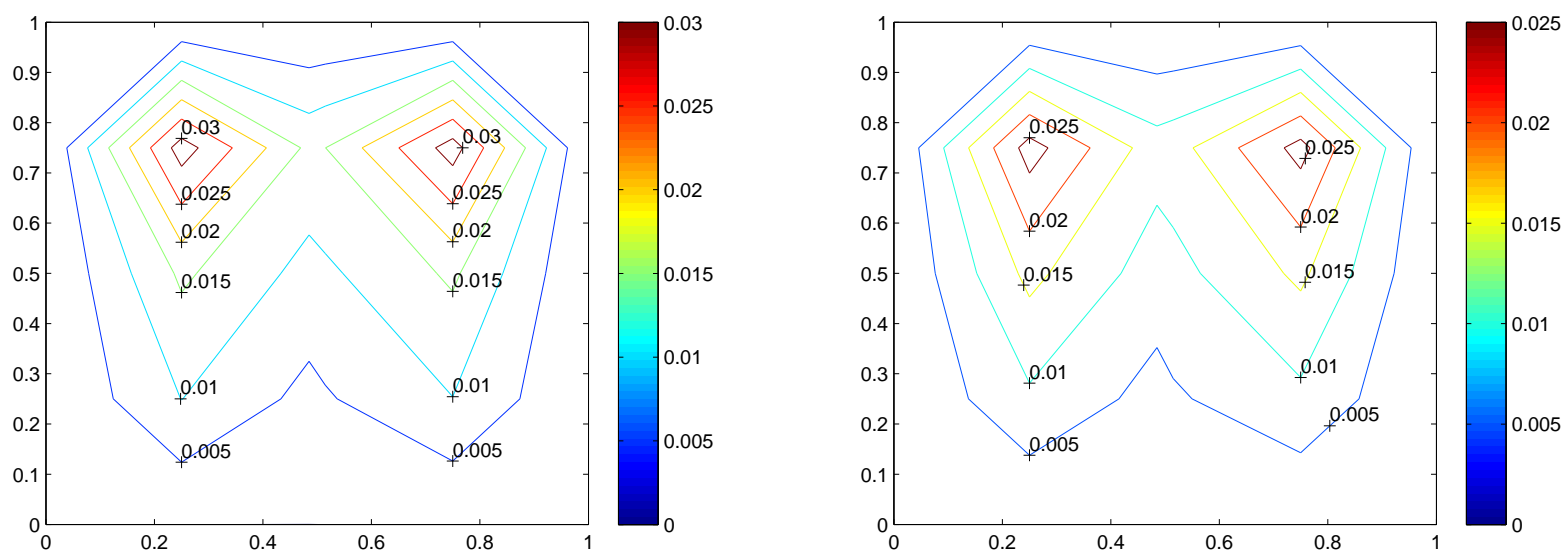

Fig. 8. Homogeneous (left) and multiscale (right) MsFEM solutions for $n=4$. 
In Figs. 8, 10, 12 the homogeneous and multiscale solutions for 4, 8 and 16 finite elements respectively are shown in isolines view. In Fig. 13 the homogeneous and multiscale solutions for 16 finite elements are shown in 3D view. As we can see, the MsFEM solution is stable and indeed converges to the homogeneous solution in the homogenization limit.
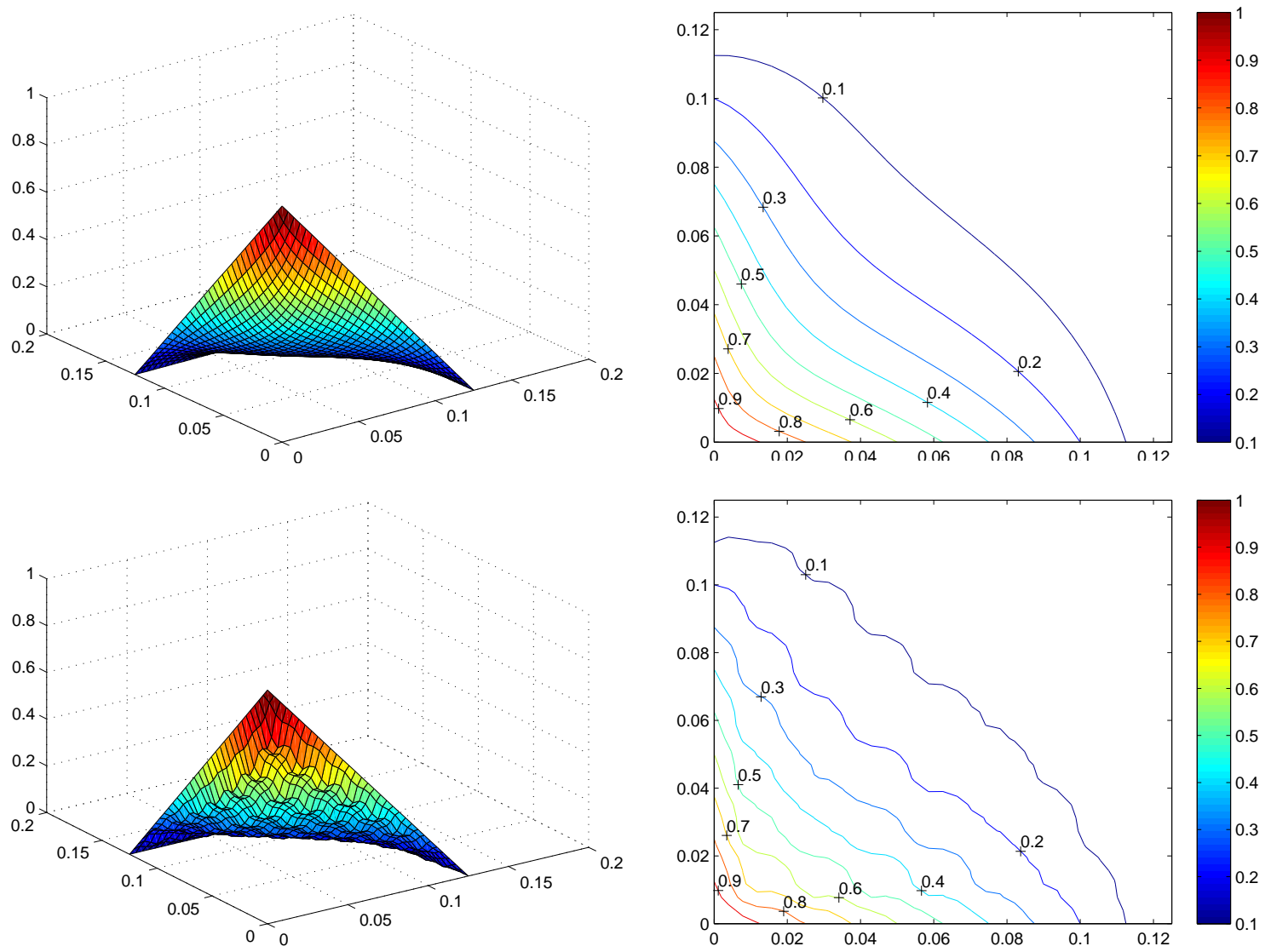

Fig. 9. Homogeneous (top) and multiscale (bottom) basis functions for $n=8$.
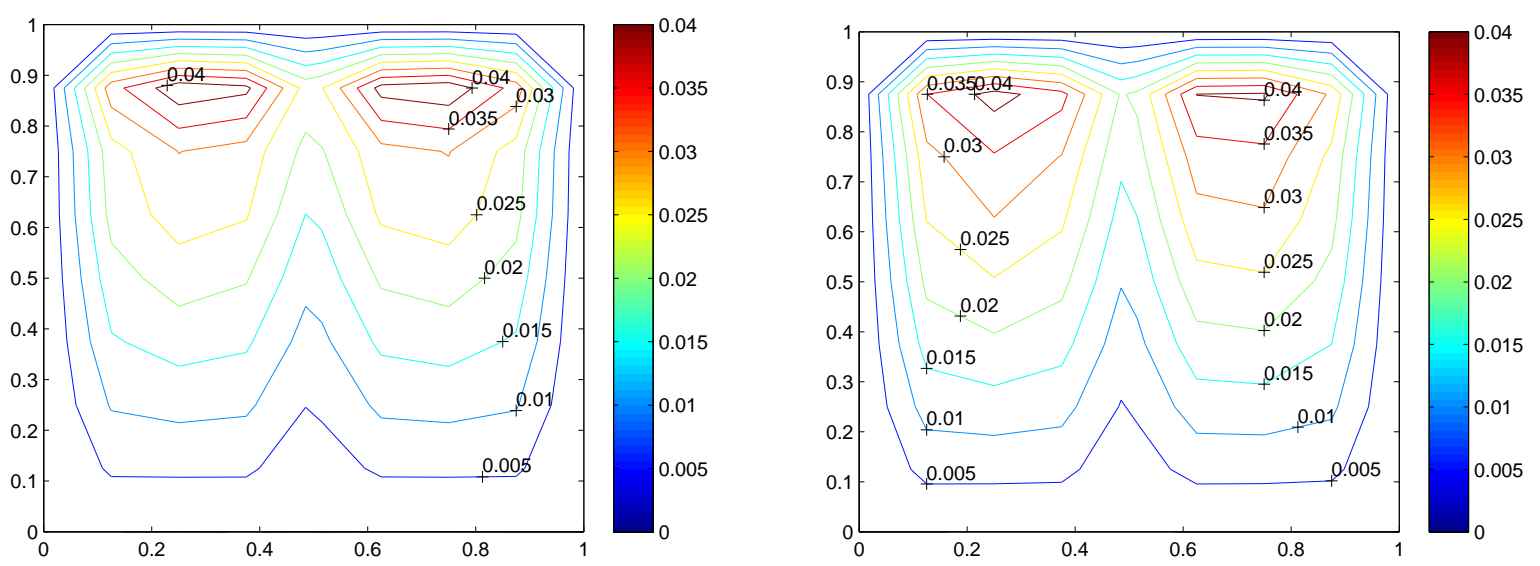

Fig. 10. Homogeneous (left) and multiscale (right) MsFEM solutions for $n=8$. 

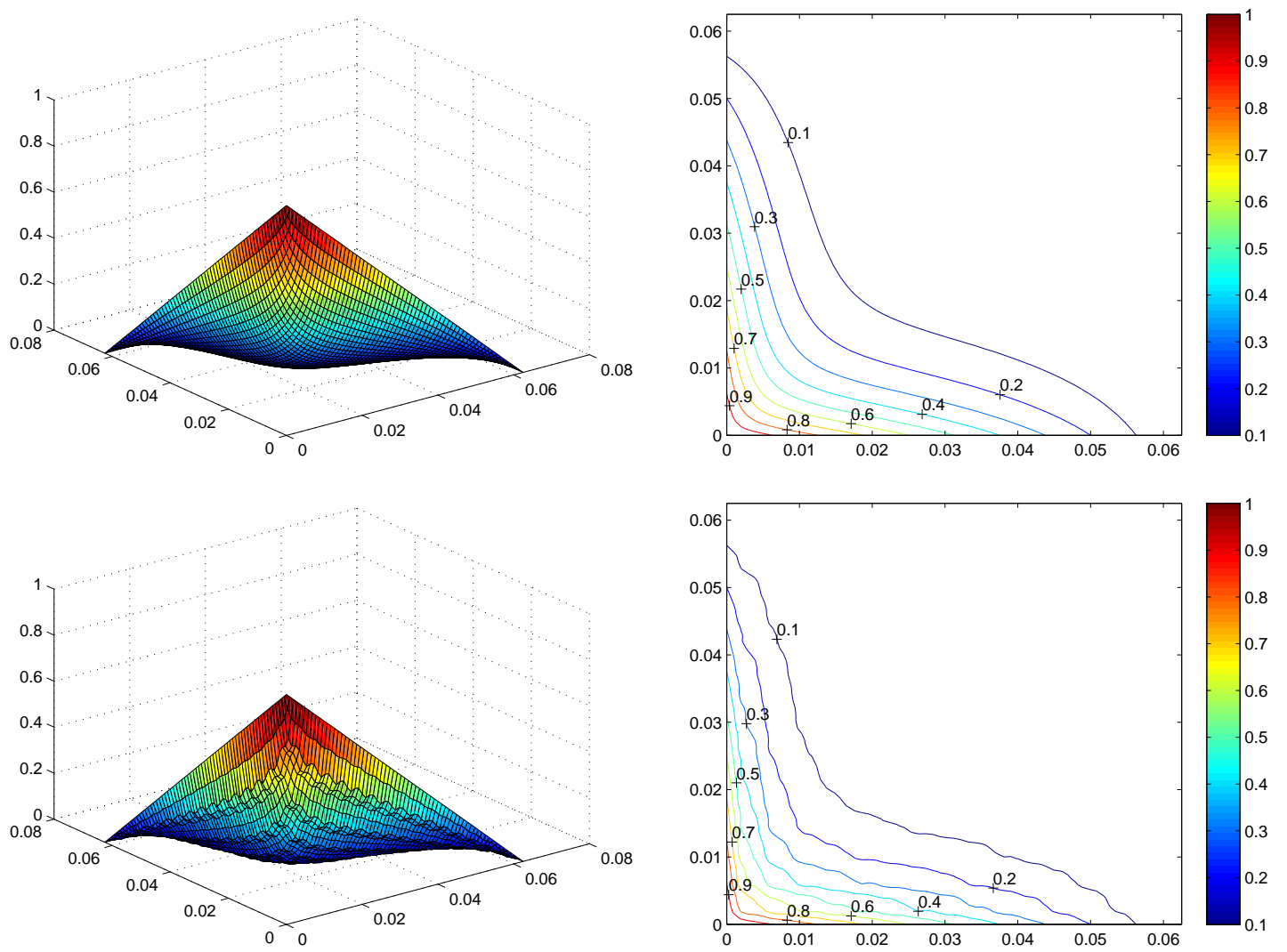

Fig. 11. Homogeneous (top) and multiscale (bottom) basis functions for $n=16$.
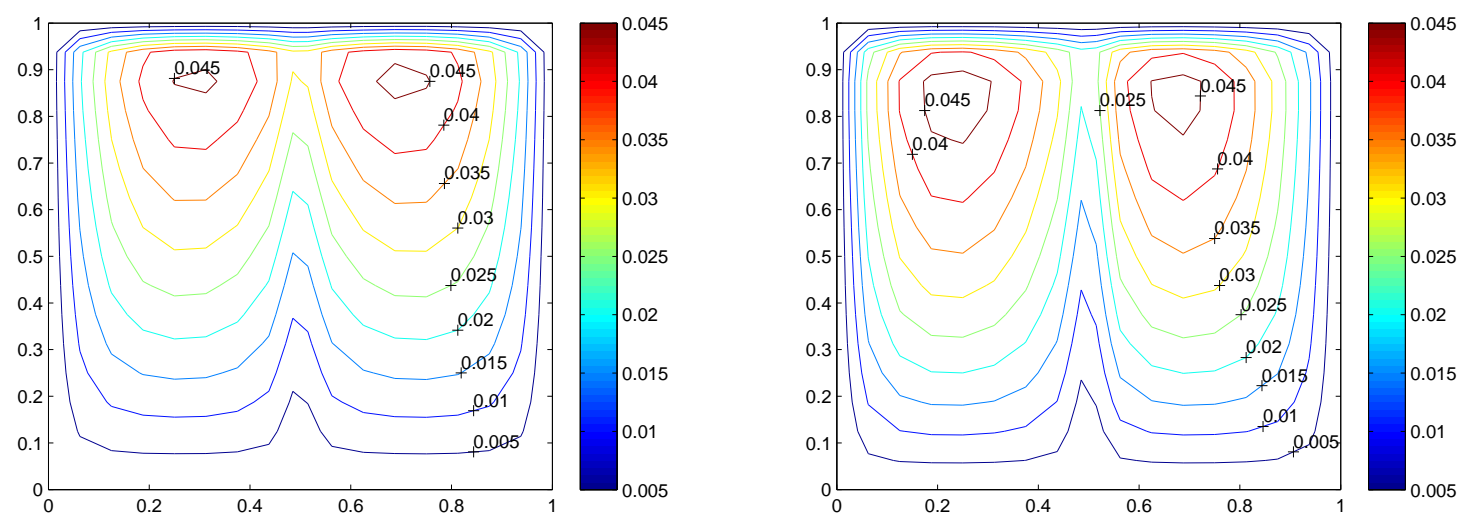

Fig. 12. Homogeneous (left) and multiscale (right) MsFEM solutions for $n=16$.
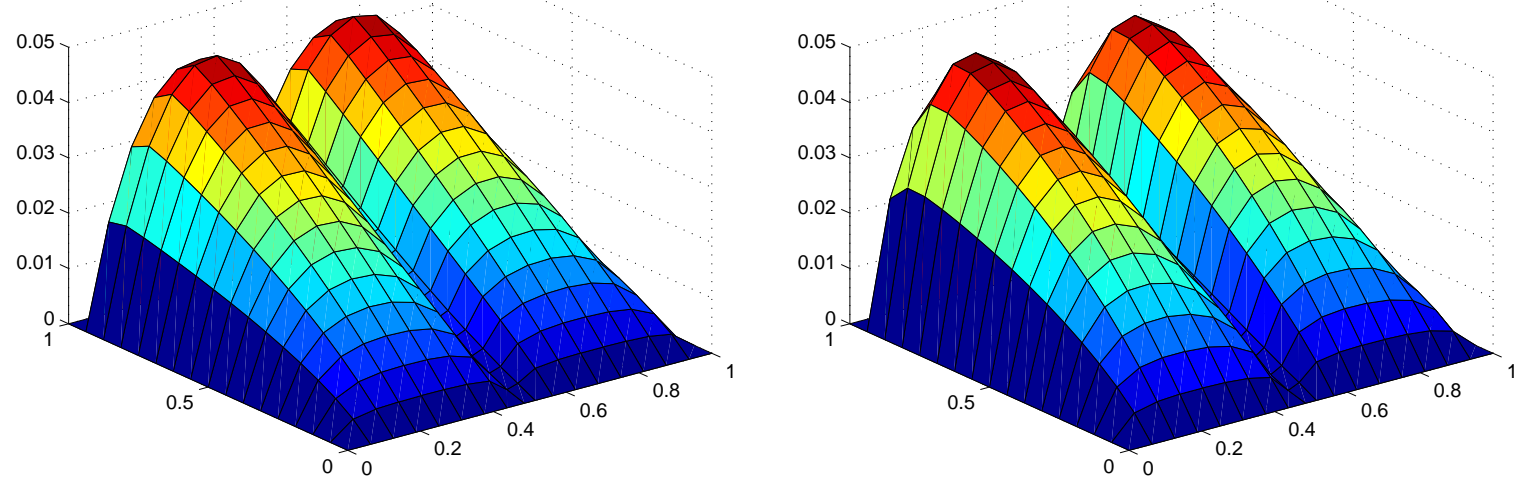

Fig. 13. Homogeneous (left) and multiscale (right) MsFEM solutions for $n=16$ in 3D view.

Mathematical Modeling and Computing, Vol. 7, No.1, pp. 146-157 (2020) 
The convergence results of the above experiments are shown in Table 1 for different norms. Here $u_{m}$ is the MsFEM solution, $u_{h}$ is the homogeneous solution,

$$
\begin{gathered}
\|u\|_{\max }=\max _{\substack{0 \leqslant i \leqslant n \\
0 \leqslant j \leqslant n}}\left|u_{i j}\right|, \quad\|u\|_{W_{2}^{(1)}}=\sqrt{\sum_{i, j=0}^{n}\left(u_{i j}^{2}+\left(\frac{\partial u_{i j}}{\partial x_{1}}\right)^{2}+\left(\frac{\partial u_{i j}}{\partial x_{2}}\right)^{2}\right)}, \\
\|u\|_{F}=\sqrt{\sum_{i, j=0}^{n} u_{i j}^{2}}, \quad\|u\|_{1}=\max _{0 \leqslant j \leqslant n} \sum_{i=0}^{n}\left|u_{i j}\right| .
\end{gathered}
$$

The obtained results confirm that the MsFEM solution converges in the homogenization limit for sufficiently large Peclet numbers.

Table 1. Convergence results.

\begin{tabular}{|c|c|c|c|c|c|c|}
\hline$n$ & $\varepsilon$ & $\frac{\varepsilon}{h}$ & $\left\|u_{m}-u_{h}\right\|_{\max }$ & $\left\|u_{m}-u_{h}\right\|_{W_{2}^{(1)}}$ & $\left\|u_{m}-u_{h}\right\|_{F}$ & $\left\|u_{m}-u_{h}\right\|_{1}$ \\
\hline 4 & 0.0625 & $\frac{1}{4}$ & 0.0141 & 0.1301 & 0.0355 & 0.0526 \\
\hline 8 & 0.0156 & $\frac{1}{8}$ & 0.0082 & 0.0708 & 0.0180 & 0.0292 \\
\hline 16 & 0.0039 & $\frac{1}{16}$ & 0.0061 & 0.0500 & 0.0129 & 0.0165 \\
\hline
\end{tabular}

\section{Conclusions}

The multiscale finite element method was applied to solving the advection-diffusion problem in the inhomogeneous medium with a thin channel. The case of large Peclet numbers and the multiscale medium was considered. It was shown that the solution obtained by MsFEM is stable and corresponds to the natural advection-diffusion process, while the FEM solution contains unnatural oscillations and does not take into account the small scale inhomogeneities. Numerical experiments confirm that the MsFEM solution converges to the homogenized one in the homogenization limit. Therefore, the multiscale finite element method is efficient for solving the formulated problem with large Peclet numbers in the multiscale medium.

[1] Savula Ya. Numerical analysis of problems of mathematical physics by variational methods. Lviv, LNU (2004), (in Ukrainian).

[2] Efendiev Y., Hou T. Multiscale finite element methods. Theory and application. New York, SpringerVerlag (2009).

[3] Hou T., Wu X., Cai Z. Convergence of a multiscale finite element method for elliptic problems with rapidly oscillating coefficients. Mathematics of Computation. 68 (227), 913-943 (1999).

[4] Spodar N., Savula Ya. Application of multiscale finite element method for solving the one-dimensional advection-diffusion problem. Physico-mathematical modelling and informational technologies. 19, 190197 (2014), (in Ukrainian).

[5] Spodar N., Savula Ya. Application of multiscale finite element method for solving the advection-diffusion problems. Visnyk of the Lviv University. Series Applied mathematics and informatics. 24, 92-100 (2016), (in Ukrainian).

[6] Spodar N., Savula Ya. Computational aspects of multiscale finite element method. Physico-mathematical modelling and informational technologies. 23, 169-177 (2016), (in Ukrainian).

[7] Mazuriak N., Savula Ya. Numerical analysis of the advection-diffusion problems in thin curvilinear channel based on multiscale finite element method. Mathematical modeling and computing. 4 (1), 59-68 (2017). 
[8] Savula Ya. H., Koukharskyi V.M., Chaplia Ye. Ya. Numerical analysis of advection-diffusion in the continuum with thin canal. Numerical Heat Transfer, Part A: Applications: An International Journal of Computation and Methodology. 33 (3), 341-351 (1998).

[9] Kukharskyy V., Kukharska N., Savula Ya. Application of Heterogeneous Mathematical Models for the Solving of Heat and Mass Transfer Problems in Environments with Thin Heterogeneties. Physico-mathematical modelling and informational technologies. 4, 132-141 (2006), (in Ukrainian).

[10] Rashevskij P. Course of differential geometry. Moscow, Leningrad, State publishing house of technical and theoretical literature (1950), (in Russian).

\title{
Числове дослідження адвекції-дифузії в неоднорідному середовищі $з$ тонким каналом різномасштабним методом скінченних елементів
}

\author{
Мазуряк Н. В., Савула Я. Г. \\ Львівський національний університет імені Івана Франка, \\ вул. Університетсъка, 1, 79000, Львів, Україна
}

Розглянуто задачу адвекції-дифузії в неоднорідному середовищі з тонким каналом. До розв'язування цієї задачі застовано різномасштабний метод скінченних елементів. Показано, що отриманий розв'язок є стійким та збіжним для достатньо великих чисел Пекле. Наведено та проаналізовано результати обчислювальних експериментів.

Ключові слова: різномасштабний метод скінченних елементів, адвекція-дифузія, неоднорідне середовище. 